\title{
Can We Evaluate the Relation between Position of Humeral Head and Tear Size, Degeneration in Rotator Cuff Tear on Magnetic Resonance Imaging?
}

\author{
Doo-Sup $\mathrm{Kim}^{1,2 \bowtie}$ \\ ${ }^{1}$ Department of Orthopedic Surgery, Yonsei University Wonju College of Medicine, ${ }^{2}$ Yonsei Institute of Sports Science and Exercise Medicine, Wonju, Korea
}

It is easy to think that rotator cuff tears clearly change the position of the center of rotation in the humeral head. ${ }^{1)}$ On the other hand, it is unclear which factor is involved. Recently, Kim and Seo "Relationship of posterior decentering of the humeral head with tear size and fatty degeneration in rotator cuff tear" (Clin Shoulder Elbow 2019;22(3):121-127) concluded that the occurrence of decentering may be related to the rotator cuff tear size, degree of subscapularis tendon tear, and fatty degeneration of the rotator cuff tendons. The interesting point in this study was that posterior decentering occurs, even at small to medium sized cuff tears. The changes in humeral head position might cause a change in the overall shoulder kinematics. ${ }^{2}$ Even small tears may be an indication of surgery to prevent scapular dyskinesis.

Another thing to note is the role of the subscapularis tendon. ${ }^{3)}$ In general, the subscapularis muscle acts as an anterior restraint in patients with shoulder instability. In this study, however, in patients with cuff tears, an increase in the degree of subscapularis tendon tears was associated with posterior translation of the humeral head. In other words, a subscapularis tendon tear appears to be involved in posterior translation, but further research is needed.

The degree of fatty degeneration has also been reported to be a critical factor in posterior decentering. In addition, severe fatty degeneration is usually associated with massive cuff tears that involve two and more tendons. Therefore, posterior decentering occurs easily in many cases of massive rotator cuff tear. ${ }^{4)}$

Importantly, not all massive rotator cuff tears in patients involve posterior translation of the humeral head. The humeral head position is centered in patients with a well-maintained force couple. Therefore, further studies for related factors will be needed.
In Yun et al.'s study, the cuff off value of the humeral head position was $2 \mathrm{~mm}$. On the other hand, it is unclear if a difference of approximately $2 \mathrm{~mm}$ is clinically meaningful. Moreover, the exclusion criteria did not include a frozen shoulder. If patients have a frozen shoulder, the magnetic resonance (MR) contrast material may not spread evenly to the anterior and posterior shoulder joint. Therefore, in Kim et al.'s study, who measured the humeral head position relative to the glenoid, patients with a frozen shoulder should be excluded.

All scans were measured at the supine position, which could be another limitation. The supine position limits the movement of the scapular. Because of this, the humeral position might have changed. In massive cuff tears, changes in the humeral head position can occur in the superior to inferior direction as well as in the anterior to posterior direction. Therefore, a research tool that can observe both directions is essential. The representative one is 2-dimensional-3-dimensional (2D-3D) shape matching technique. ${ }^{6}$

Magnetic resonance imaging (MRI) for an evaluation of the humeral position provides information on the soft tissue, but it is also a static measurement tool that cannot provide the dynamic status. Recently, open MRI has been in the spotlight, but this can take images only at limited shoulder elevation angles. A 2D-3D image registration technique would be better for measuring the in vivo kinematic-values than MRI.

\section{References}

1. Updegrove GF, Armstrong AD, Mosher TJ, Kim HM. Posterior displacement of supraspinatus central tendon observed on magnetic resonance imaging: a useful preoperative

\section{Correspondence to: Doo-Sup Kim}

Department of Orthopedic Surgery, Yonsei University Wonju College of Medicine, 20 Ilsan-ro, Wonju 26426, Korea

Tel: +82-33-741-1356, Fax: +82-33-741-1357, E-mail: dskim1974@hanmail.net, ORCID: https://orcid.org/0000-0002-9025-085X

Editorial does not need an IRB approval.

Financial support: None. Conflict of interests: None. 
indicator of rotator cuff tear characteristics. Arthroscopy. 2015;31(11):2089-98. doi: 10.1016/j.arthro.2015.04.096.

2. Cutti AG, Veeger HE. Shoulder biomechanics: today's consensus and tomorrow's perspectives. Med Biol Eng Comput. 2009;47(5):463-6. doi: 10.1007/s11517-009-0487-3.

3. Yoo JC, Rhee YG, Shin SJ, et al. Subscapularis tendon tear classification based on 3-dimensional anatomic footprint: a cadaveric and prospective clinical observational study. Arthroscopy. 2015;31(1):19-28. doi: 10.1016/j.arthro.2014.08.015.

4. Parsons IM, Apreleva M, Fu FH, Woo SL. The effect of rotator cuff tears on reaction forces at the glenohumeral joint.
J Orthop Res. 2002;20(3):439-46. doi: 10.1016/S07360266(01)00137-1.

5. Yun G, Kang Y, Ahn JM, et al. Posterior decentering of the humeral head on shoulder MR arthrography: significant association with posterior synovial proliferation. AJR Am J Roentgenol. 2017;208(6):1297-303. doi: 10.2214/AJR.16.17198.

6. Banks SA, Hodge WA. Accurate measurement of threedimensional knee replacement kinematics using single-plane fluoroscopy. IEEE Trans Biomed Eng. 1996;43(6):638-49. doi: 10.1109/10.495283. 